\title{
Suprachoroidal gas injection as a complication of pars plana vitrectomy confirmed by computed tomography
}

This article was published in the following Dove Press journal:

Clinical Ophthalmology

29 March 2012

Number of times this article has been viewed

\author{
Akihito Uji \\ Department of Ophthalmology and \\ Visual Sciences, Kyoto University \\ Graduate School of Medicine, Kyoto, \\ Japan
}

Background: Suprachoroidal gas injection has been reported as a complication of pneumatic retinopexy, and the usefulness of B-scan ultrasonography has been reported. A case of suprachoroidal gas injection as a complication of pars plana vitrectomy confirmed by computed tomography $(\mathrm{CT})$ is presented here.

Methods: A 64-year-old woman developed a vitreous hemorrhage due to a retinal tear secondary to an old branch retinal vein occlusion. A 23-gauge pars plana vitrectomy was performed. Sclerotomies were performed at a $30^{\circ}$ angle. After air-fluid exchange, sulfur hexafluoride gas was injected through a temporo-superior sutured sclerotomy with direct visualization of the entire needle tip. Postoperatively, a large choroidal elevation was identified temporo-superiorly with intravitreal gas, and the patient complained of a mild headache.

Results: On postoperative Day 7, a CT scan showed two low-density areas, confirming the presence of suprachoroidal gas; intravitreal gas blocked visualization of the suprachoroidal gas on B-scan ultrasonography. On postoperative Day 14, the intravitreal gas resolved and a CT scan showed a small amount of residual suprachoroidal gas. By this time, the headache had completely resolved.

Conclusion: Suprachoroidal gas injection is a rare complication of pars plana vitrectomy that resolves spontaneously. In this case, CT scans confirmed the presence of suprachoroidal gas despite the presence of intravitreal gas.

Keywords: suprachoroidal gas, complication, pars plana vitrectomy, computed tomography

\section{Introduction}

Baker and Hainsworth ${ }^{1}$ first reported suprachoroidal gas injection as a complication of pneumatic retinopexy. They theorized that the cause of the complication was excessive withdrawal of the needle and that B-scan ultrasonography was useful for confirming the presence of suprachoroidal gas. However, because intravitreal gas reflects ultrasound waves, B-scan ultrasonography is not useful for imaging retina and choroid in cases with intravitreal gas. Here, a case of suprachoroidal and intravitreal gas injected without excessive needle withdrawal is reported, confirmed by computed tomography (CT), as a complication of pars plana vitrectomy.

Correspondence: Akihito Uji

Department of Ophthalmology and Visual Sciences, Kyoto University

Graduate School of Medicine,

54 Shogoin Kawahara-cho, Sakyo-ku,

Kyoto 606-8507, Japan

$\mathrm{Tel}+8|7575| 3248$

Fax +8I 757520933

Email akihitol@kuhp.kyoto-u.ac.jp

\section{Case report}

A 64-year-old woman developed a vitreous hemorrhage due to a retinal tear nasosuperior to the optic nerve head and secondary to an old branch retinal vein occlusion. Her visual acuity was 20/20. A 23-gauge pars plana vitrectomy was performed using the EdgePlus ${ }^{\circledR}$ Trocar System (Alcon Laboratories Inc, Fort Worth, TX) after 
phacoemulsification cataract extraction. Sclerotomies were created at a $30^{\circ}$ angle parallel to the limbus. Core vitrectomy was performed followed by peripheral vitrectomy without shaving the vitreous base. Scleral indentation of the periphery was performed to detect any peripheral breaks. Although no associated retinal detachment was seen intraoperatively, an air-fluid exchange was performed after photocoagulation of the retinal tear. At the end of surgery, the trocars were removed, all sclerotomies were sutured with 8.0 Vicryl* (Ethicon Inc, Novartis Animal Health Inc, Basel, Switzerland) and $1.5 \mathrm{~mL}$ of $100 \%$ sulfur hexafluoride (SF6) gas was injected through a temporo-superior sutured sclerotomy with direct visualization of the entire needle tip. When the needle was withdrawn and the internal cylinder was pressed to maintain intraocular pressure (IOP), the patient experienced severe ocular pain for a minute.

On the first postoperative day, a large choroidal elevation was temporo-superiorly identified with borderline intravitreal gas detected below the lower retinal vascular arcade. Visual acuity was counting fingers. The IOP was $15 \mathrm{mmHg}$ and the patient complained of a mild headache that was controlled with acetaminophen. On postoperative Day 7, a CT scan showed two separate low-density areas, which confirmed the presence of suprachoroidal gas; the gas was not visible on B-scan ultrasonography (Figure 1) because of the presence of intravitreal gas (Figure 2A-C). Visual acuity was 20/40. On postoperative Day 10, the intravitreal gas filled $<10 \%$ of the vitreous cavity and the suprachoroidal gas

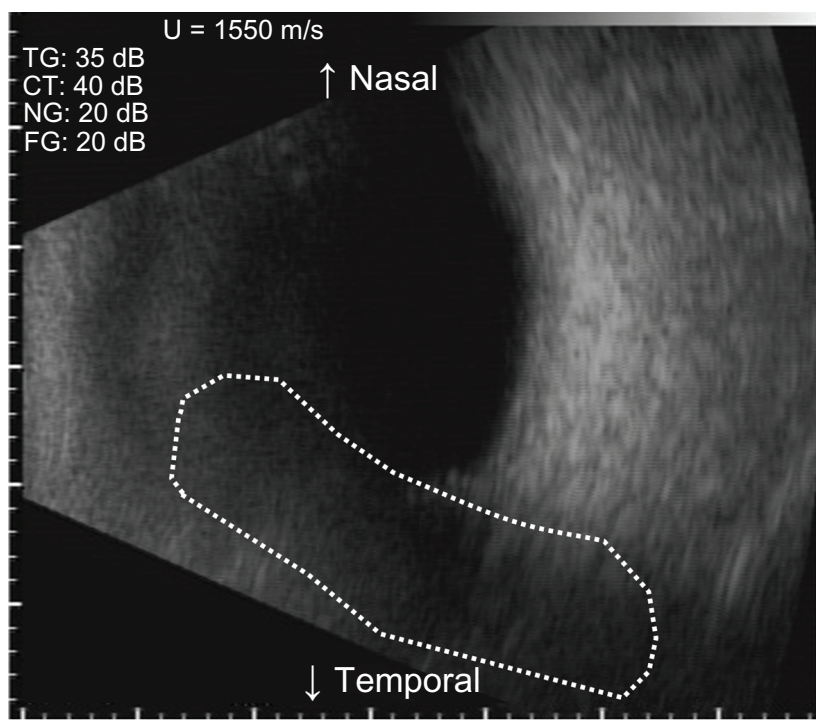

Figure I Horizontal scan of a B-scan ultrasonography image of intravitreal gas and choroid elevation with the patient seated on postoperative Day 7. Because of the reflection of the intravitreal gas (dotted outline), B-scan ultrasonography could not image the choroidal elevation, which developed superior to the ocular fundus. started to diminish (Figure 3A). Visual acuity was 20/20. By postoperative Day 14, the intravitreal gas had resolved and the CT scans showed a small amount of residual suprachoroidal gas (Figure 2D-F); further, the headache had resolved completely, and visual acuity was 20/20. The retina was attached and, 8 months later, visual acuity was 20/20 (Figure 3B).

\section{Discussion}

To date, various complications of pars plana vitrectomy have been reported, including endophthalmitis, suprachoroidal hemorrhage, hypotony, retinal tears, and choroidal detachments. ${ }^{2-6}$ Although iatrogenic suprachoroidal gas injection has been previously reported as a complication of pneumatic retinopexy, ${ }^{1,7}$ to the best of our knowledge, only one study has described suprachoroidal SF6 gas injection as a complication of 23 -gauge vitrectomy resulting from intraoperative retraction of the infusion cannula. ${ }^{8}$ Here, the natural course and CT confirmation and follow-up of suprachoroidal SF6 gas injected during pars plana vitrectomy is presented.

Because a choroidal elevation resulting from gas injection appears similar to that of an expulsive choroidal hemorrhage, earlier diagnosis of this complication using $\mathrm{CT}$ may reduce the associated stress for surgeons and patients. In the current case, the presence of suprachoroidal gas was confirmed using CT instead of B-scan ultrasonography. Because intravitreal gas reflects ultrasound waves, B-scan ultrasonography could not image the choroidal elevation, which developed superior to the ocular fundus when the patient was supine and seated (Figure 1). In contrast, a CT showed well-circumscribed low-density areas, confirming the presence of suprachoroidal gas (Figure 2). However, rightfully, radiation exposure is an important consideration when $\mathrm{CT}$ is used. Thus, CT usage should be minimized to avoid unnecessary exposure to radiation.

The patient experienced ocular pain during withdrawal of the needle and a persistent postoperative headache. The pain might have been caused by the suprachoroidal gas, as previously reported by Jabaly-Habib et al. ${ }^{7}$ Because the pain was mild and controllable in the current case, the gas was not removed and the pain diminished with time.

Because gas was injected with direct visualization of the entire needle tip, the cause of the suprachoroidal gas injection might have been the withdrawal of the needle with pressure placed on the internal cylinder. Although this maneuver does not usually cause this complication, it was speculated that ciliary body elevation resulting from insertion of the needle through the sclerotomy, which was created at a $30^{\circ}$ angle, 

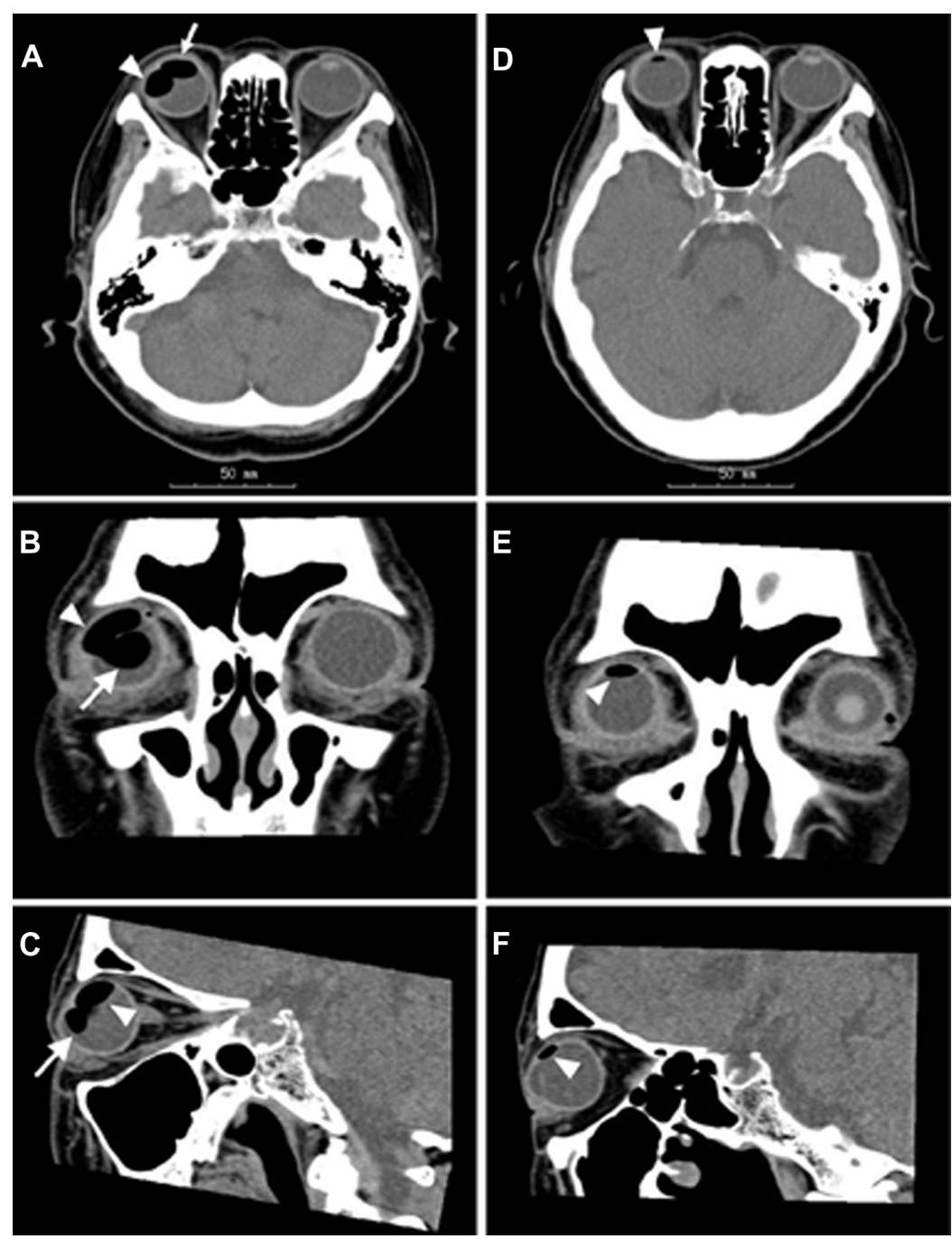

Figure 2 CT scans showing the presence of suprachoroidal gas. (A-C) On postoperative Day 7, two separate low-density areas of suprachoroidal gas (arrows) and intravitreal gas (arrowheads) are seen. (D-F) On postoperative Day 14, the intravitreal gas has resolved and a small amount of residual suprachoroidal gas is seen (arrowheads). Abbreviation: $\mathrm{CT}$, computed tomography.

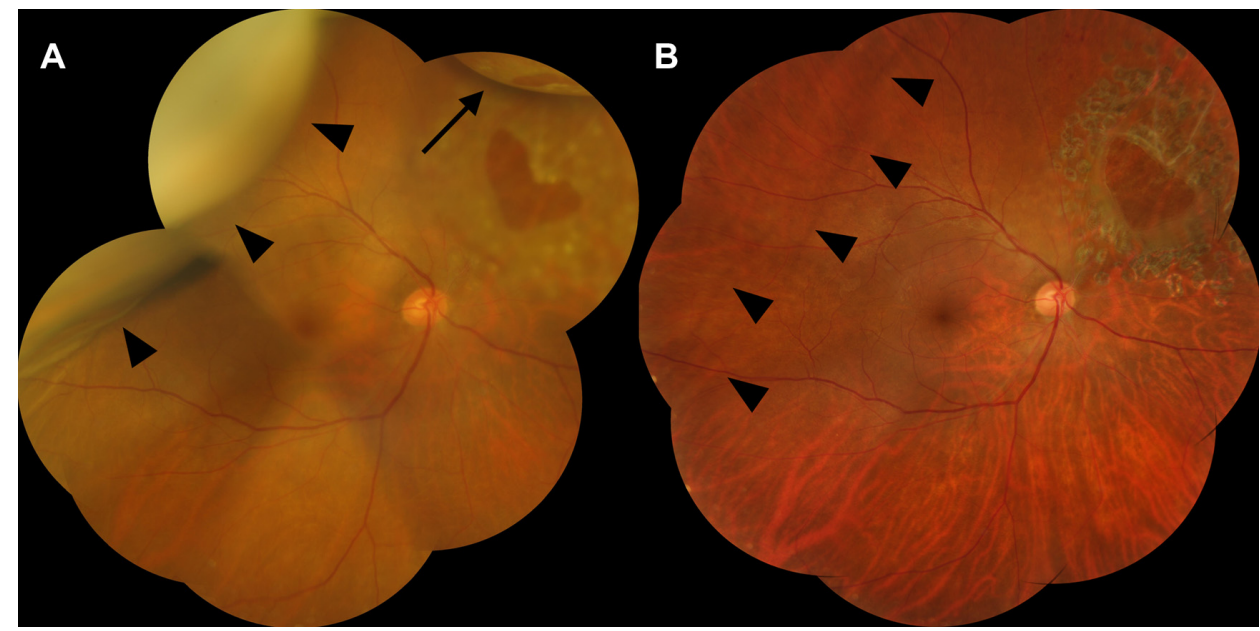

Figure 3 (A) Color fundus photograph of suprachoroidal gas. On postoperative Day 10, a large, temporo-superior choroidal elevation (arrowheads) is seen and the intravitreal gas (arrow) occupies $<10 \%$ of the vitreous cavity. (B) The retina is attached, and 8 months later, the visual acuity is 20/20. The choroidal folds (arrowheads) persist long after the suprachoroidal gas has resolved. 
induced the suprachoroidal gas injection at the time of needle withdrawal. This may be preventable by injecting the gas through a site other than the sclerotomy.

\section{Conclusion}

Suprachoroidal gas injection is a rare complication of pars plana vitrectomy that resolves spontaneously. CT is useful for confirming the presence of suprachoroidal gas even when intravitreal gas is present.

\section{Disclosure}

The author did not receive any financial support for this study and has no financial interest in the subject matter.

\section{References}

1. Baker SR, Hainsworth DP. Suprachoroidal gas as a complication of pneumatic retinopexy. Retina. 2000;20(2):224-225.
2. Albrieux M, Rouberol F, Bernheim D, Romanet JP, Chiquet C. Comparative study of 23 -gauge vitrectomy versus 20 -gauge vitrectomy for the treatment of rhegmatogenous retinal detachment. Graefes Arch Clin Exp Ophthalmol. 2011;249(10):1459-1468.

3. Chieh JJ, Rogers AH, Wiegand TW, Baumal CR, Reichel E, Duker JS. Short-term safety of 23-gauge single-step transconjunctival vitrectomy surgery. Retina. 2009;29(10):1486-1490.

4. Gupta OP, Ho AC, Kaiser PK, et al. Short-term outcomes of 23-gauge pars plana vitrectomy. Am J Ophthalmol. 2008;146(2):193-197.

5. Ooto S, Kimura D, Itoi K, et al. Suprachoroidal fluid as a complication of 23-gauge vitreous surgery. Br J Ophthalmol. 2008;92(10):1433-1434.

6. Parolini B, Romanelli F, Prigione G, Pertile G. Incidence of endophthalmitis in a large series of 23-gauge and 20-gauge transconjunctival pars plana vitrectomy. Graefes Arch Clin Exp Ophthalmol. 2009;247(7):895-898.

7. Jabaly-Habib HY, Fineberg EM, Tornambe PE, Lang YZ, Garzozi HJ. Prolonged pain following unintentional injection of gas into the suprachoroidal space during pneumatic retinopexy. Retina. 2003;23(5):722-723.

8. Tarantola RM, Folk JC, Shah SS, et al. Intraoperative choroidal detachment during 23-gauge vitrectomy. Retina. 2011;31(5):893-901.
Clinical Ophthalmology

\section{Publish your work in this journal}

Clinical Ophthalmology is an international, peer-reviewed journal covering all subspecialties within ophthalmology. Key topics include: Optometry; Visual science; Pharmacology and drug therapy in eye diseases; Basic Sciences; Primary and Secondary eye care; Patient Safety and Quality of Care Improvements. This journal is indexed on

Submit your manuscript here: http://www.dovepress.com/clinical-ophthalmology-journal

\section{Dovepress}

PubMed Central and CAS, and is the official journal of The Society of Clinical Ophthalmology (SCO). The manuscript management system is completely online and includes a very quick and fair peer-review system, which is all easy to use. Visit http://www.dovepress.com/ testimonials.php to read real quotes from published authors. 